\title{
CHI3L1 alleviate acute liver injury by inhibiting Th1 cells differentiation through STAT3 signaling pathway
}

\author{
Shaopeng Zhang", Xinzheng Dai", Yong Shi, Xiaowen Zhu, Yongjiu Dai, Xiaofeng Qian, Jian Gu \\ Liver Transplantation Center, First Affiliated Hospital, Nanjing Medical University, Nanjing, China \\ Contributions: (I) Conception and design: J Gu, X Qian, S Zhang; (II) Administrative support: J Gu, X Qian, X Dai; (III) Provision of study materials \\ or patients: S Zhang, X Dai, Y Shi, X Zhu, Y Dai; (IV) Collection and assembly of data: J Gu, S Zhang, Y Shi; (V) Data analysis and interpretation: X \\ Zhu, Y Dai; (VI) Manuscript writing: All authors; (VII) Final approval of manuscript: All authors. \\ \#These authors contributed equally to this work. \\ Correspondence to: Jian Gu; Xiaofeng Qian. Liver Transplantation Center, First Affiliated Hospital, Nanjing Medical University, Nanjing 210029 , \\ China. Email: gujian@njmu.edu.cn; qianxiaofeng@njmu.edu.cn.
}

Background: Acute liver injury (ALI) is a severe liver disease. Chitinase 3-like-1 (CHI3L1), a protein belonging to the glycosyl hydrolase family 18, is involved in many diseases, such as inflammatory diseases, bacterial infections, and various malignant tumors; however, the function of CHI3L1 in ALI remains unclear. The objective of this study was to evaluate the protective functions of CHI3L1 against thioacetamide (TAA)induced ALI in mice and explore its potential mechanisms.

Methods: Data from 20 patients with ALI and 10 healthy subjects was collected. Serum CHI3L1, serum aspartate transaminase (AST), and serum alanine aminotransferase (ALT) were measured. To establish ALI mouse models, thioacetamide was intraperitoneally injected into groups of the CHI3L1-knockout (CHI3L1$\mathrm{KO})$ and wild-type (WT) mice (80 and $150 \mathrm{mg} / \mathrm{kg}$ ). Recombinant CHI3L1 protein (rCHI3L1) $(5 \mu \mathrm{g} / \mathrm{kg})$, IFN- $\gamma(500 \mathrm{ng}$ ), and WP1033 (an inhibitor of P-STAT3, $0.2 \mathrm{~mL}$ ) were injected before TAA treatment, after which the effects were estimated. Splenic CD4+CD62L+ naive T cells were isolated from CHI3L1-KO mice and stimulated to differentiate into regulatory T (Treg) cells, T-helper 1 (Th1) cells, T-helper 2 (Th2) cells, and T-helper 17 (Th17) cells.

Results: Increased serum CHI3L1 levels were seen both in healthy subjects and post-therapy patients compared with ALI patients. CHI3L1 levels were negatively correlated with serum ALT and AST levels in ALI patients. CHI3L1-KO group showed higher serum ALT and AST levels than the WT group following TAA treatment, while tail vein injection of $\mathrm{rCHI} 3 \mathrm{~L} 1$ reduced liver tissue injury and improved Treg cell differentiation in vivo. In vitro experiment showed that knockout of CHI3L1 improved IFN- $\gamma+$ Th1 cell differentiation. Furthermore, intraperitoneal administration of IFN- $\gamma$ produced more severe hepatocellular necrosis compared with rCHI3L1 injection alone. Mechanism study showed that T-box expressed in T cells (T-bet), and signal transducer and activator of transcription 3 (STAT3), play a critical role in adversely mediating the effect of CHI3L1, which is consistent with the finding that treatment with WP1033 downregulated the differentiation of the Th1 cells in vitro and reduced severity of liver injury in vivo.

Conclusions: CHI3L1 reduced the production of IFN- $\gamma$ and inhibited Th1 cell differentiation through the STAT3 signaling pathway, which could be a potential therapeutic strategy for treating ALI.

Keywords: Acute liver injury (ALI); chitinase 3-like-1 (CHI3L1); Th1 cell; signal transducer and activator of transcription 3 (STAT3)

Submitted Aug 26, 2020. Accepted for publication Jan 10, 2021.

doi: 10.21037/atm-20-6127

View this article at: http://dx.doi.org/10.21037/atm-20-6127

(c) Annals of Translational Medicine. All rights reserved. 


\section{Introduction}

Acute liver injury (ALI) is a severe condition characterized by widespread hepatotoxicity and hepatocellular necrosis that is drug-induced, viral, or caused by autoimmune hepatitis (1-3). Acetaminophen (APAP) is a common cause of drug-related hepatotoxicity-either through accidental or intentional overdose-and can induce serious liver injury and failure (4). Thioacetamide (TAA) is a widely-used hepatotoxicant in laboratory studies due to its ability to elicit a pathophysiological process that causes liver damage in animals (5). Compared with other reagents, TAA exhibits several advantages: TAA has higher reproducibility, higher reliability for various species, and lower mortality. Also, TAA mimics a longer damage-inducing latency, similar to that in humans (6). Therefore, TAA was used to establish ALI mice models in this study.

There is increasing evidence that innate and adaptive immune responses play a crucial role in the causation and acceleration of liver injury over a short period. For innate immune reaction, neutrophils, which migrate from sinusoids into the parenchyma, generate excessive reactive oxygen, accumulating in the liver tissue, causing hepatocyte injury (7). Kupffer cells are activated by increased phagocytic activity and increased proinflammatory cytokine formation, including tumor necrosis factor $\alpha$ (TNF- $\alpha$ ) and interleukin-6 (IL-6) (8). In the early stages, local adaptive immune activity predominantly depends on the accumulation of $\mathrm{CD}^{+} \mathrm{T}$ lymphocytes along with the release of high quantities of proinflammatory cytokines, such as interferon (IFN)- $\gamma$ and interleukin-17 (IL-17) (9). Furthermore, regulatory T (Treg) cells can maintain immune homeostasis by suppressing $\mathrm{CD}^{+} \mathrm{T}$ cells. Accordingly, the ratio of Treg/T-helper (Th) cells was closely related to the pathogenesis of ALI caused by infiltrating Th cells; however, the exact underlying mechanisms are not well-understood $(10,11)$.

Chitinase 3-like-1 (CHI3L1), known in mice as BRP39 and humans as YKL-40, is found on chromosome 1 and 2 in each species. CHI3L1 can induce the synthesis of $39-\mathrm{kDa}$ protein lacking chitinase activity (12). Various cells-including hepatocytes, macrophages, chondrocytes, and hepatic stellate cells-can express and secrete CHI3L1. One of the main functions of CHI3L1 appears to be the down-regulation of proinflammatory cytokine expression (13). Studies have also shown that CHI3L1 is associated with cell regeneration, cell survival, cell invasion, and promoting angiogenesis $(14,15)$. Furthermore, CHI3L1 plays an essential role in disease pathogenesis, such as viral respiratory infection and hepatic fibrosis, where circulating levels of CHI3L1 are increased. Recent studies have reported that patients with fibrosis, chronic hepatitis $\mathrm{B}$, and chronic hepatitis $\mathrm{C}$ have higher levels of $\mathrm{CHI} 3 \mathrm{~L} 1$ compared with healthy individuals $(16,17)$.

Despite this, the role of CHI3L1 in ALI is not wellunderstood. Based on existing literature, we hypothesized that CHI3L1 might be associated with T lymphocytes' regulation and involved in the protection of ALI. To investigate this hypothesis, serum CHI3L1 levels were measured in healthy subjects, liver injury patients, and posttherapy patients. Furthermore, serum ALT and AST levels were detected in TAA-induced ALI in wild-type (WT), CHI3L1-knockout (CHI3L1-KO), and rCHI3L1-injected mice. We found that knockout of $\mathrm{CHI} 3 \mathrm{~L} 1$ resulted in more severe liver injury, whereas treatment with $\mathrm{rCHI} 3 \mathrm{~L} 1$ injection attenuated TAA-induced liver injury and decreased serum ALT and AST levels significantly by restraining inflammatory cell infiltration.

Moreover, the rCHI3L1 treatment increased the proportion of Foxp $3^{+}$Treg cells, and reduced $\mathrm{CD} 4^{+} \mathrm{IFN}-\gamma^{+}$ $\mathrm{T}$ cells. IFN- $\gamma$ treatment aggravated the liver injury, while expression of transcription factor T-box expressed in T cells (T-bet), and phosphorylated signal transducer and activator of transcription 3 (P-STAT3) were upregulated in CHI3L1$\mathrm{KO}$ group. Signal transducer and activator of transcription 3 (STAT3) inhibitor alleviated liver injury. These results suggest that CHI3L1 could play a therapeutic role in reducing ALI through inhibition of $\mathrm{CD} 4^{+} \mathrm{IFN}-\gamma^{+} \mathrm{Th} 1$ cell-related immune response by suppressing the T-bet/STAT3 pathway.

The following article is presented following the ARRIVE reporting checklist (available at http://dx.doi.org/10.21037/ atm-20-6127).

\section{Methods}

\section{Study subjects}

20 patients from the first Affiliated Hospital of Nanjing Medical University were recruited if they had been diagnosed ALI. The causes included acute viral hepatitis, drug damage, and poisoning (10 patients with acute viral hepatitis, 8 patients with drug damage and 2 patients with poisoning). All the patients and healthy subjects (age from 29-54 years old) don't suffer from chronic diseases such as hypertension and diabetes or other immune-related diseases, and we excluded acute exacerbations of chronic 
Table 1 Scores used to evaluate the severity of liver damage (18)

\begin{tabular}{|c|c|c|c|}
\hline Numerical assessment & Congestion & Vacuolization & Necrosis \\
\hline 1 & Minimal & Minimal & Single-cell necrosis \\
\hline 2 & Mild & Mild & $-30 \%$ \\
\hline 3 & Moderate & Moderate & $-60 \%$ \\
\hline
\end{tabular}

liver disease and acute or chronic liver failure. All subjects had a clear clinical diagnosis of ALI and were hospitalized from 2016.9-2017.9. All the patients had been treated with regular hepatoprotective drugs such as Acetylcysteine, Diammonium Glycyrrhizinate. The study was conducted in accordance with the Declaration of Helsinki (as revised in 2013). The study was approved by the Institutional Review Board of the First Affiliated Hospital of Nanjing Medical University (approval number 2015-SRFA-095), and informed consent was obtained from all participants.

\section{Measurements of serum CHI3L1, AST and ALT}

Blood was collected from healthy subjects, post-therapy patients, and ALI patients. The collected blood is separated by a centrifuge $(3,000 \times \mathrm{g}$ for 8 minutes $)$, and then the CHI3L1, ALT and AST in the serum is measured by a biochemical analyzer (AU480, Beckman Coulter, CA, USA). For mice, serum was collected from different groups following TAA induced injury, serum AST, CHI3L1 and ALT were determined with the use of a biochemical analyzer.

\section{Animals}

Wild-type (WT) C57BL/6 mice (8-12 weeks old, male, 180-220 g) and CHI3L1-knockout (CHI3L1-KO) mice (C57BL/6 background, 8-12 weeks old, male, 180-220 g) were obtained from the Animal Core Facility of Nanjing Medical University (Nanjing, China). The mice were housed in a pathogen-free animal facility with controlled temperature, humidity and 12-hour light/dark cycles. The mice were fed with sterilized water and food ad libitum. The animal experiments in the study were approved by the Institutional Animal Care and Use Committees at Nanjing Medical University (number NMU08-092), in compliance with the US National Institutes of Health guidelines for the care and use of animals.
In order to establish ALI mouse models, TAA (Sigma, Saint Louis, MO, USA) was dissolved in normal saline and injected intraperitoneally three times a week at a dose of 80 and $150 \mathrm{mg} / \mathrm{kg}$ for two weeks ( $\mathrm{n}=5$, per group). The same volume of normal saline was intraperitoneally injected into the control mice. The mice were killed $24 \mathrm{~h}$ after final treatment of TAA. Blood samples and liver specimens were collected. Tissues were preserved in liquid nitrogen. Samples from the dissected livers were fixed in $10 \%$ neutral buffered formalin for further experiments. The rCHI3L1 group was injected with rCHI3L1 (BD Biosciences, San Jose, CA, USA) by tail vein at a dose of $5 \mu \mathrm{g} / \mathrm{kg}$ before TAA treatment $(\mathrm{n}=5)$. For the IFN- $\gamma$ group, intraperitoneal administration of IFN- $\gamma$ (Sigma, Saint Louis, MO, USA) at a dose of $500 \mathrm{ng}$ each was performed before TAA injection $(\mathrm{n}=5)$. WP1033 (Sigma, Saint Louis, MO, USA) was dissolved in normal saline followed by injected via tail vein at a dose of $0.2 \mathrm{~mL}$ per mouse $(\mathrm{n}=5)$ before TAA injection. All the mice were randomly divided into groups. Finally, the mice were euthanized following an IACUC approved protocol after the experiment.

\section{Histological analysis}

The obtained liver specimen was cut into small pieces, then fixed with $4 \%$ neutral-buffered paraformaldehyde fix solution (Beyotime Biotechnology Co., Ltd., China) for one day. Then the fixed tissues were embedded in paraffin. Hematoxylin \& eosin (H\&E, Staining Kit, Beyotime Biotechnology Co., Ltd., China) staining were performed on samples of $4 \mu \mathrm{m}$ thickness. Histological analysis was performed under the light microscopy. Sections of liver samples were evaluated by an excellent pathology, and the degree of liver damage was analyzed using the Suzuki score (18). Hepatocyte necrosis, sinusoidal congestion and balloon degeneration are included in the score (see Table 1 for details). 
Table 2 List of primer used for real-time quantitative polymerase chain-reaction (RT-PCR)

\begin{tabular}{lll}
\hline Gene & Forward primer & Reverse primer \\
\hline CHI3L1 & GAGGAAGTTGCCTAGCAGTATAAT & CTGCTAGGCAACTTCCTCAGATTT \\
Foxp3 & ACCATTGGTTTACTCGCATGT & TCCACTCGCACAAAGCACTT \\
STAT3 & CAATACCATTGACCTGCCGAT & AGGAATCGGCTATATTGCTGGT \\
HPRT & TCAGTCAACGGGGGACATAAA & GGGGCTGTACTGCTTAACCAG \\
\hline
\end{tabular}

CHI3L1, Chitinase 3-like-1; Foxp3, Forkhead box P3; STAT-3, signal transducer and activator of transcription 3; HPRT, Hypoxanthine guanine phosphoribosyl transferase.

\section{Flow cytometry}

Anti-mouse CD4, CD25 antibodies (BioLegend, San Diego, CA, USA) were used to stain cells, which were analyzed by flow cytometry. Intracellular staining for Foxp3, after staining with surface antibodies, the cells were fixed/permeabilized in cytofix and permeabilization solution (BioLegend, San Diego, CA, USA) and then incubated with anti-mouse-Foxp3 (BioLegend, San Diego, CA, USA). According to the protocol, cells were stimulated with antigen and brefeldin $\mathrm{A}$, and cultured in $5 \% \mathrm{CO}_{2}$ at $37^{\circ} \mathrm{C}$ for $4-6$ hours to measure intracellular cytokines. Staining for IFN- $\gamma$, IL-17, IL-4 and tumor necrosis factor- $\alpha(\mathrm{TNF}-\alpha)$ was performed after the fixation and permeabilization using staining kit reagents (all from BioLegend, San Diego, CA, USA). Control antibodies used included Alexa Flour 647 Rat IgG2b, APC/cy7 Rat IgG2b and APC Rat IgG1 (BioLegend, San Diego, CA, USA). These experiments had been repeated three times.

\section{Induction of cells}

Magnetic selection was used to isolate Splenic CD $4^{+} \mathrm{CD} 62 \mathrm{~L}^{+}$naive $\mathrm{T}$ cells from $\mathrm{CHI} 3 \mathrm{~L} 1-\mathrm{KO}$ mice $\left(\mathrm{CD} 4^{+} \mathrm{CD} 62 \mathrm{~L}^{+} \mathrm{T}\right.$ cell isolation kit, Milteny Biotec). After that, cells were washed with culture medium. To stimulate cells, the anti-CD3/CD28 beads (Invitrogen, Carlsbad, $\mathrm{CA})$ were utilized at a bead/T cell ratio of $1: 3$ and various kinds of recombinant cytokines. Cells were stimulated to differentiate into Th1 cells by supplementation with IL-12 $(25 \mathrm{ng} / \mathrm{mL})$ plus anti-IL-4 antibody $(10 \mu \mathrm{g} / \mathrm{mL})$, or into Th2 cells by supplementation with IL-4 $(25 \mathrm{ng} / \mathrm{mL})$ plus anti-IFN- $\gamma$ antibody $(10 \mu \mathrm{g} / \mathrm{mL})$, or into Th17 cells by supplementation with transforming growth factor- $\beta$ (TGF- $\beta ; 5 \mathrm{ng} / \mathrm{mL})$ and IL-6 $(25 \mathrm{ng} / \mathrm{mL})$ in the presence of anti-IFN- $\gamma$ antibody $(10 \mu \mathrm{g} / \mathrm{mL})$ and anti-IL-4 antibody $(10 \mu \mathrm{g} / \mathrm{mL})$, or into Treg cells by supplementation with
TGF- $\beta$ (50 ng/mL) plus IL-2 (50 IU/mL). The IL-12, IL4, TGF- $\beta$, IL-6, IL-2 were purchased from R\&D Systems (Shanghai, China) and the other reagents were obtained from BD Biosciences (San Jose, CA, USA).

\section{$R N A$ isolation and quantitative real-time PCR}

Following the manufacturer's protocol, total ribonucleic acid (RNA) in liver tissues was extracted with the TRIzol kit (Invitrogen, California, USA). Then cDNA is obtained by reverse transcription using a reverse transcriptase kit (Takara Bio, Shiga, Japan). The cDNA after reverse transcription was subjected to real-time quantitative polymerase chain reaction (PCR) on the 7500 real-time PCR system (Applied Biosystems, Foster City, CA, USA). Cycling conditions consisted of denaturation of the $30 \mathrm{~s}$ at $95^{\circ} \mathrm{C}$, combined annealing of $20 \mathrm{~s}$ at $60{ }^{\circ} \mathrm{C}$, and extension of $30 \mathrm{~s}$ at $72{ }^{\circ} \mathrm{C}$ by 40 cycles. The expression of target genes was normalized to $\beta$-ACTIN as control. All experiments had been done in triplicates. Primers used were shown in Table 2.

\section{Western blot}

A small piece of liver tissue is quickly placed in the RIPA lysis buffer (Beyotime Biotechnology Co., Ltd., China) and homogenized with a homogenizer. The buffer was placed on ice for 30 minutes and vortexed every 10 minutes. Then the solution was centrifuged at $12,000 \times \mathrm{g}$ for 10 minutes at $4{ }^{\circ} \mathrm{C}$, and the concentration of the extracted protein was measured by a BCA kit assay (Beyotime Biotechnology Co., Ltd., China). A total of $20 \mathrm{mg}$ of protein was separated by the SDS-PAGE gel electrophoresis, and then was transferred onto nitrocellulose membranes (Sunshine Biotechnology, China). The PVDF membranes were blocked with skim milk powder at $25^{\circ} \mathrm{C}$ for 2 hours. After that, these membranes were incubated with rabbit antibodies to STAT3 (Abcam Hongkong, China ab119352), 

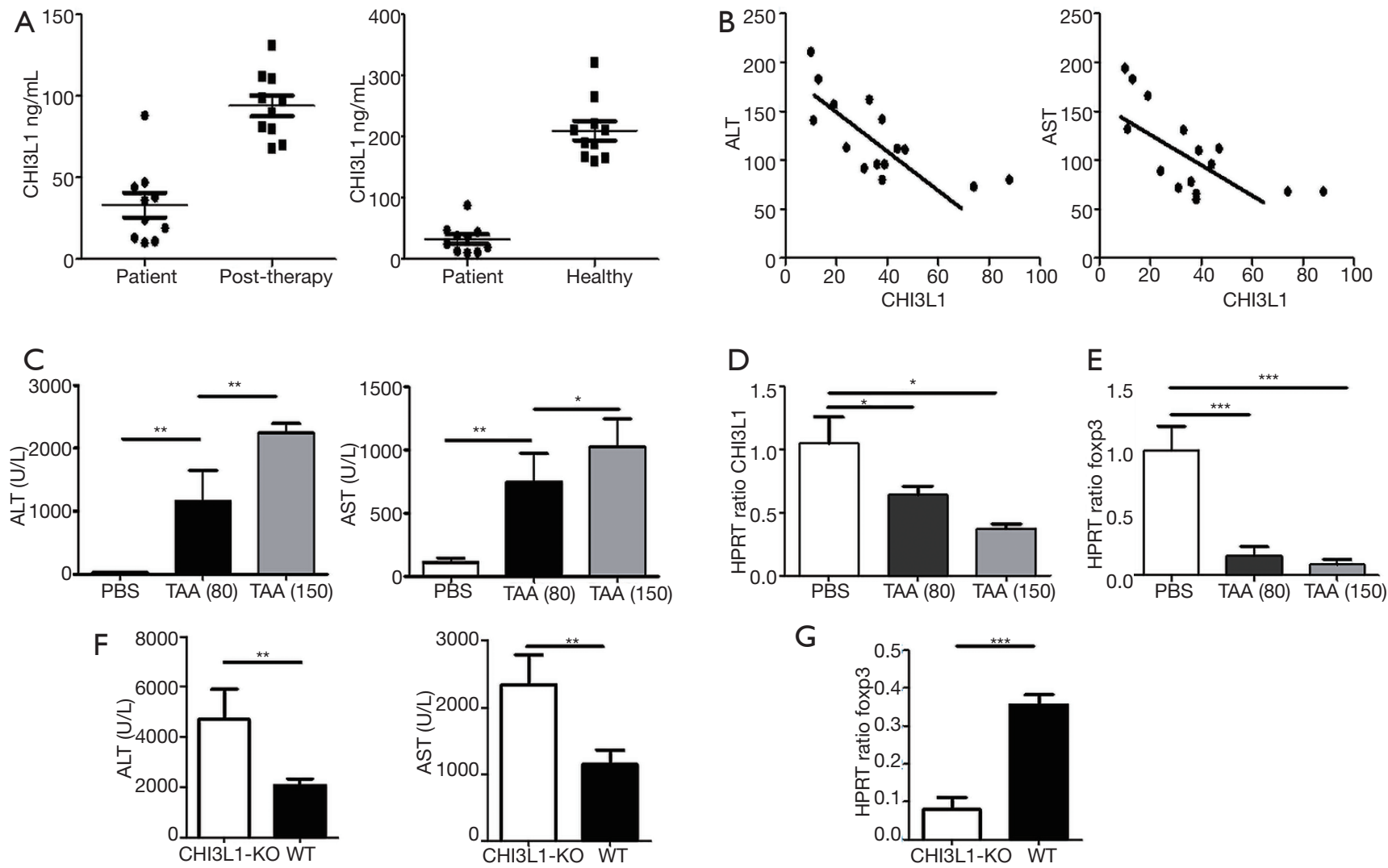

Figure 1 Negative correlation between CHI3L1 and liver injury level. To investigate the relationship between CHI3L1 and liver injury, serum from 10 patients with ALI and 10 health volunteers were derived and analyzed. (A) The levels of CHI3L1 in patients before and posttherapy, as well as that in healthy volunteers, were detected through ELISA. (B) The correlation of ALT and AST to CHI3L1 in the serum of patients with ALI were shown. We also confirmed the result in the murine model. (C) The serum ALT, AST levels of the murine ALI models with the treatment of PBS or different doses of TAA were shown. The level of mRNA expression of CHI3L1 (D) and Foxp3 (E) was determined by RT-PCR. The serum ALT, AST levels (F), and liver tissues expression of Foxp3 mRNA (G) of the ALI models in WT and CHI3L1-KO mice. Data are shown as means \pm SEM from 3 independent experiments, and each group include 5 mice. ${ }^{* *}, \mathrm{P}<0.01 ;$ ***, $\mathrm{P}<0.001$. Abbreviations: CHI3L1, Chitinase 3-like-1; ALI, acute liver injury; ALT, alanine aminotransferase; AST, aspartate transaminase; TAA, thioacetamide; Foxp3, forkhead box P3; WT, wild-type; CHI3L1-KO, CHI3L1-knockout.

P-STAT3 (Abcam Hongkong, China ab76315) and $\beta$-actin (Abcam Hongkong, China ab8226) overnight.

After washing 3 times with TBST buffer, these membranes were incubated with HRP-conjugated goat antirabbit IgG (Santa Cruz Biotechnology, Santa Cruz, CA) at $25^{\circ} \mathrm{C}$ for 2 hours. Proteins were detected and visualized by using ECL detection system (Alpha Innotech, CA).

\section{Statistical analyses}

Data analysis was performed using GraphPad Prism 7.0 software. Parametric testing between the two groups was performed via unpaired $t$-tests. To compare parametric testing among three or more groups, one-way ANOVA was used. $\mathrm{P} \leq 0.05$ was considered statistically significant.

\section{Results}

\section{The negative correlation between CHI3L1 and liver injury level}

To evaluate the effect of CHI3L1 in ALI, the expression of CHI3L1 and ALT and AST in serum were detected. CHI3L1 levels differed greatly in ALI patients pre- and post-therapy (Figure 1A). CHI3L1 was dramatically increased in 10 post-therapy patients and 10 healthy 
A

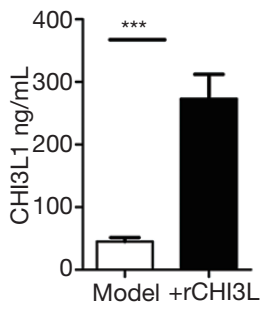

B

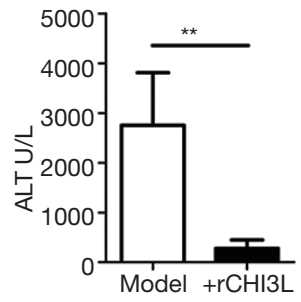

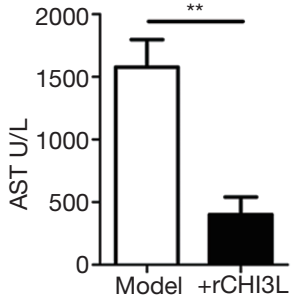

C

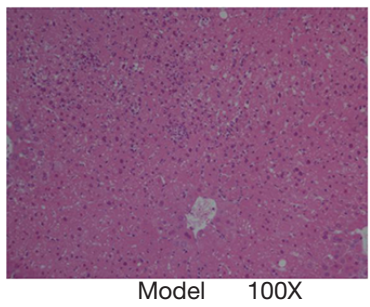

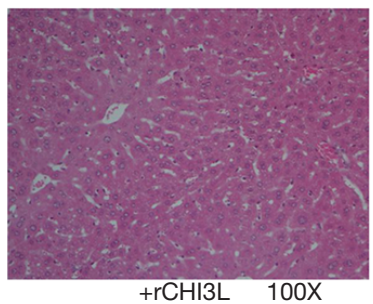

D
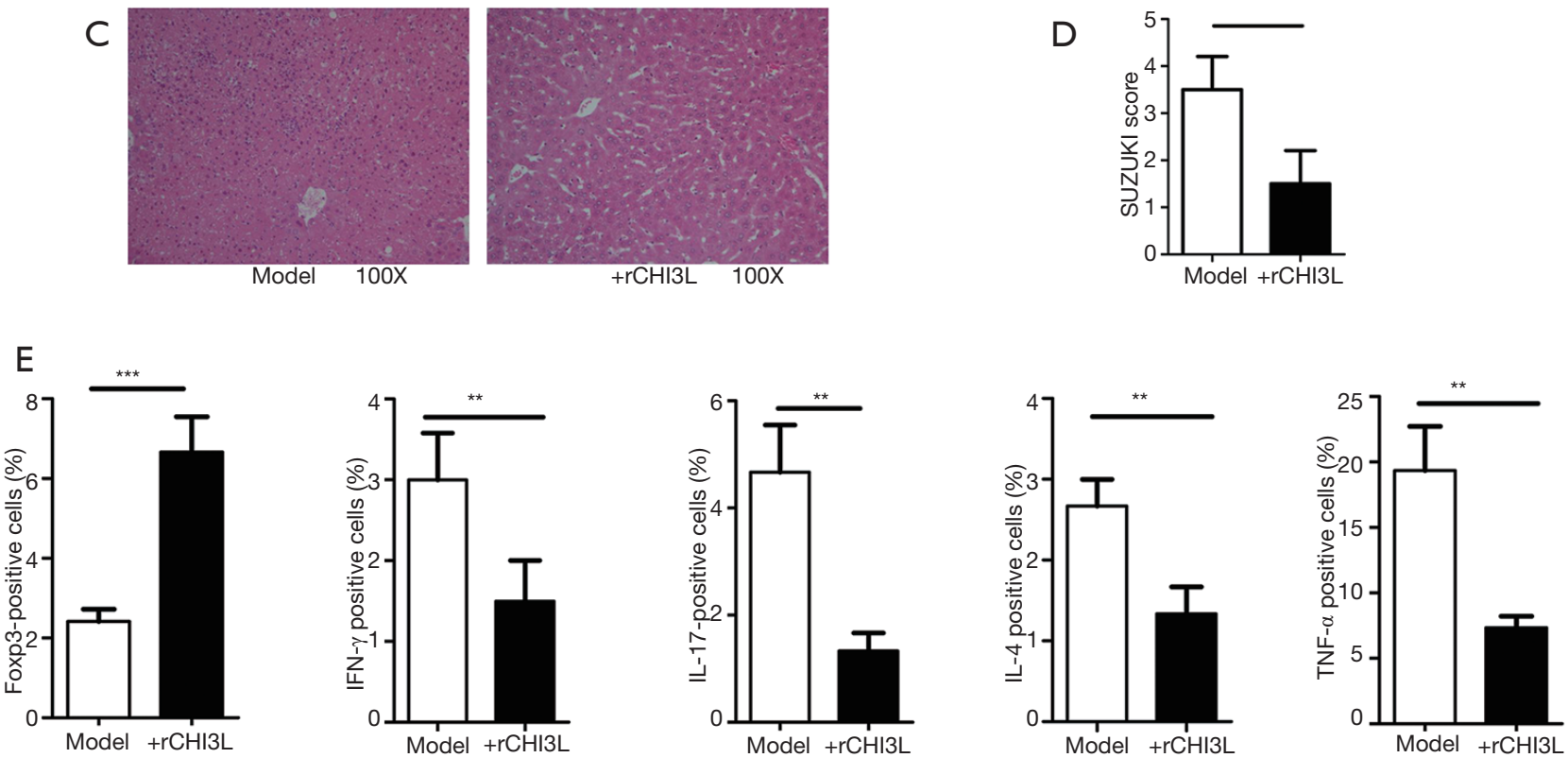

Figure 2 rCHI3L1 reduces TAA-induced liver injury in mice. rCHI3L1 was injected through the tail vein followed TAA injection to verify the function of CHI3L1 in the liver. (A) The levels of CHI3L1 in the serum of the ALI murine model were determined through ELISA. (B) The serum ALT, AST levels of ALI models with or without the treatment of rCHI3L1 were shown. (C) Histological changes following the ALI are shown (H\&E staining, original magnification, $\times 100$ ). (D) Suzuki scores were measured as mentioned above. (E) The proportion of IL-4, Foxp3, IL-17, IFN- $\gamma$, and TNF- $\alpha$ positive cells was determined by flow cytometry. Data are shown as means \pm SEM from 3 independent experiments, and each group include 5 mice. ${ }^{* *}, \mathrm{P}<0.01$; ${ }^{* *}, \mathrm{P}<0.001$. Abbreviations: $\mathrm{rCHI} 3 \mathrm{~L} 1$, recombinant $\mathrm{CHI} 3 \mathrm{~L} 1$ protein; IL-4, interleukin-4; IL-17, interleukin-17; IFN- $\gamma$, interferon- $\gamma$; TNF- $\alpha$, tumor necrosis factor- $\alpha$.

subjects compared to 10 ALI patients, and CHI3L1 exhibited a negative correlation with ALT levels accordingly (Figure 1B). To support the effect of CHI3L1 in ALI, ALT and AST serum levels and CHI3L1 were investigated in murine models with TAA-induced liver injury. ALT and AST levels were dramatically increased relative to TAA dosage, while CHI3L1 mRNA expression was reduced (Figure 1C,D). Interestingly, the mRNA levels of Foxp3 in the liver were markedly decreased after the injection of TAA (Figure 1E). Furthermore, ALT and AST levels in $\mathrm{CHI} 3 \mathrm{L1}^{-/}$mice were higher than WT models, while Foxp3 was significantly inhibited in $\mathrm{CHI} 3 \mathrm{~L}^{-/-}$mice (Figure $1 F, G$ ).

\section{rCHI3L1 reduces TAA-induced liver injury in mice}

Before TAA treatment, rCHI3L1 was injected by tail vein, and the serum ALT and AST levels of the WT mice and the rCHI3L1-treated mice were detected. The expression of CHI3L1 in mice injected with $\mathrm{rCHI} 3 \mathrm{~L} 1$ was significantly higher than that in the WT mice (Figure 2A). As expected, serum ALT and AST levels in the rCHI3L1-treated mice were significantly lower compared to WT littermates (Figure 2B). To further investigate the protective effect of $\mathrm{rCHI} 3 \mathrm{~L} 1$ in ALI, histological results were compared between $\mathrm{rCHI} 3 \mathrm{~L} 1$ mice and their WT littermates (Figure $2 C, D$ ) and suggested that $\mathrm{rCHI} 3 \mathrm{~L} 1$ reversed TAA- 
A

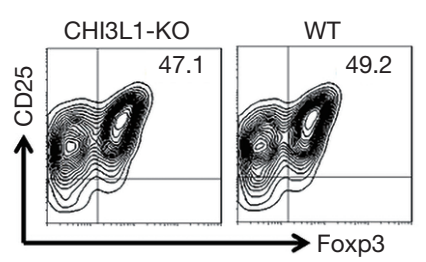

C
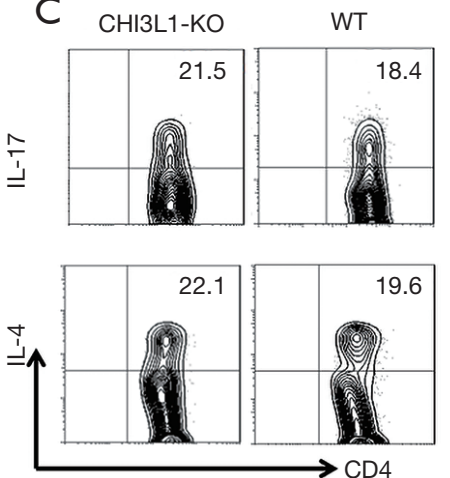

D

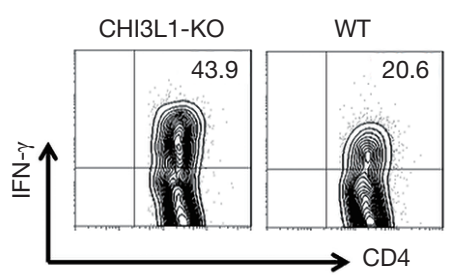

NS

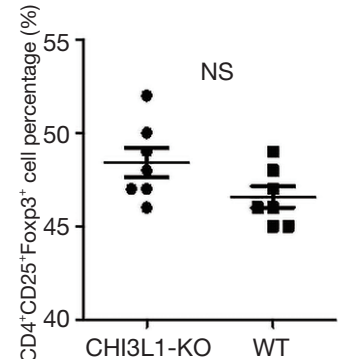

$\mathrm{E}$

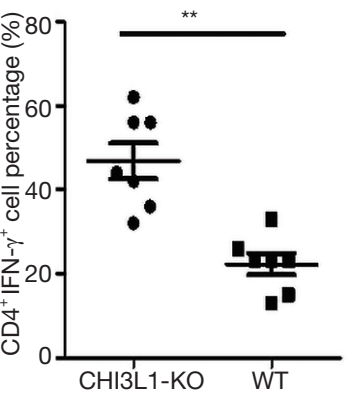

Figure $3 \mathrm{CHI} 3 \mathrm{~L} 1$ specifically decreased the differentiation of $\mathrm{CD}^{+} \mathrm{INF}-\gamma^{+} \mathrm{T}$ cells. To determine whether the differentiation of CD4+ $\mathrm{T}$ cells subpopulation was mediated by $\mathrm{CHI} 3 \mathrm{~L} 1, \mathrm{CD} 4^{+} \mathrm{CD} 62^{+}$naive $\mathrm{T}$ cells were isolated from the WT mice and $\mathrm{CHI} 3 \mathrm{~L} 1^{-/-}$mice by magnetic beads, and a translation experiment was performed. Representative contour plots (A) and statistic result (B) for Foxp3 expression during iTregs induction in the treatment of TGF- $\beta$. Representative contour plots (C) for IL-17 and IL-4 expression during Th17 and Th2 induction. Representative contour plots (D) and statistic result (E) for IFN- $\gamma$ expression during Th1 induction. Data are shown as means \pm SEM from 5 independent experiments. NS, no significant; ${ }^{* *}, \mathrm{P}<0.01$. Abbreviations: CD4, cluster of differentiation 4; CD62, cluster of differentiation 62; iTregs, induced regulatory T cells; TGF- $\beta$, transforming growth factor-beta; Th17, T-helper 17; Th2, T-helper 2; Th1, T-helper 1.

induced liver injury. To determine the main subsets that contributed to the regulative ability of $\mathrm{CHI} 3 \mathrm{~L} 1$, measures of Foxp $3^{+}, \mathrm{INF}-\gamma^{+}, \mathrm{IL}-17^{+}, \mathrm{IL}-4^{+}$, and $\mathrm{TNF}-\alpha^{+}$in $\mathrm{CD} 4^{+}$ $\mathrm{T}$ cells were taken after TAA treatment. Remarkably, an increased ratio of Foxp $3^{+} \mathrm{T}$ cells was found in rCHI3L1treated mice. Furthermore, reduced amounts of other proinflammatory $\mathrm{T}$ cells were found in $\mathrm{rCHI} 3 \mathrm{~L} 1$-treated mice (Figure 2E).

\section{CHI3L1 specifically decreased the differentiation of $C D 4^{+}$ INF- $\gamma^{+}$T cells}

To determine whether CHI3L1 mediated the differentiation of $\mathrm{CD}^{+} \mathrm{T}$ cells subpopulation, $\mathrm{CD} 4^{+} \mathrm{CD} 62^{+}$naive $\mathrm{T}$ cells were isolated from the WT mice and $\mathrm{CHI} 3 \mathrm{~L}^{-/-}$ mice by magnetic beads, and a translation experiment was performed. There was no appreciable difference in the percentage of $\mathrm{CD}^{+} \mathrm{CD}_{25} 5^{+} \mathrm{Foxp}^{+} \mathrm{T}$ cells in $\mathrm{CHI} 3 \mathrm{~L}^{-/-}$ mice compared with their WT littermates at different doses of TGF- $\beta$, regardless of the rCHI3L1 treatment (Figure $3 A, B$ ). No marked differences in the proportions of Th2 and Th17 cells were observed between the CHI3L $1^{-/-}$ mice and their WT littermates after co-culture with various cytokines (Figure 3C); however, between the two groups, $\mathrm{CHI} 3 \mathrm{~L}^{-/-}$mice-derived naive $\mathrm{T}$ cells were more liable to transfer into INF- $\gamma^{+}$Th1 cells, indicating that $\mathrm{CHI} 3 \mathrm{~L} 1$ may alleviate the injury of ALI by influencing the differentiation of Th1 cells (Figure 3D,E).

\section{INF- $\gamma$ aggravated liver injury even with the addition of $r$ CHI3L1}

Given previous findings of decreased INF- $\gamma$ expression following $\mathrm{rCHI} 3 \mathrm{~L} 1$ treatment and enhanced INF- $\gamma$ levels in CHI3L1-KO, it was hypothesized that CHI3L1 might mediate INF- $\gamma$ effects on liver injury. We found that $\mathrm{rCHI} 3 \mathrm{~L} 1$ treatment significantly improved CHI3L1 production, both with and without the addition of INF- $\gamma$ 


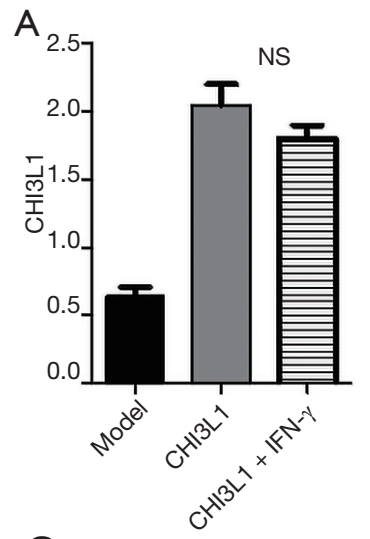

C

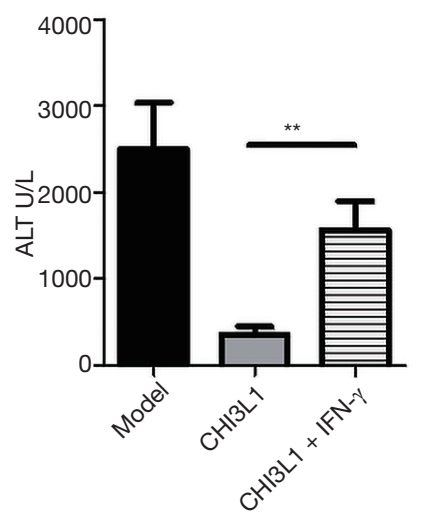

B
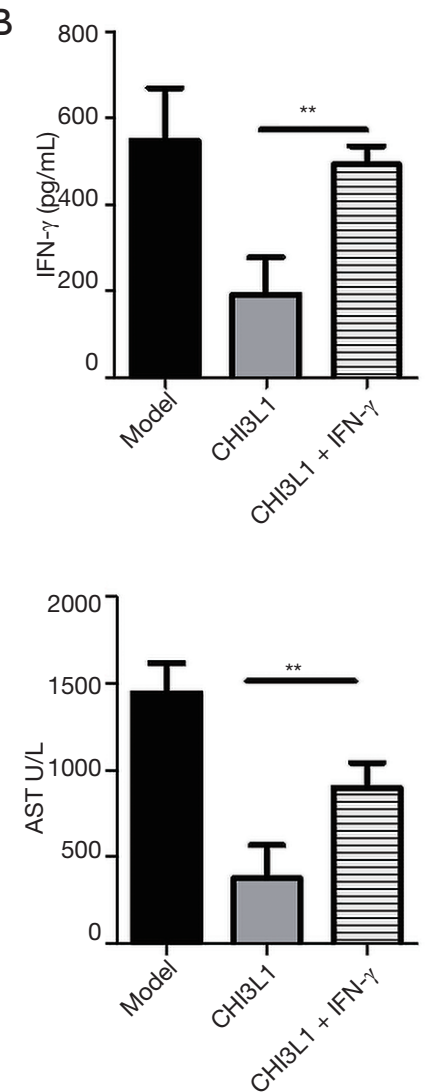

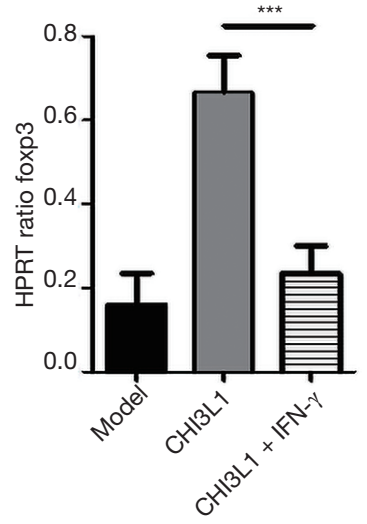

D

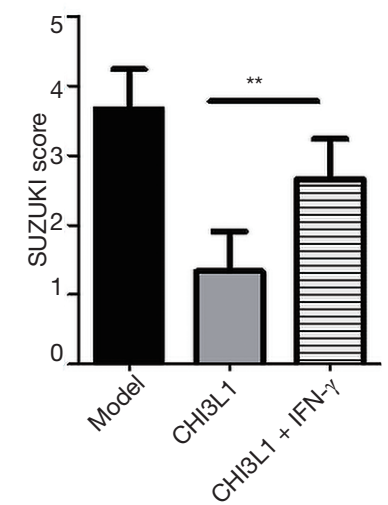

Figure 4 INF- $\gamma$ aggravated liver injury even with the addition of rCHI3L1. rCHI3L1 and INF- $\gamma$ were injected through the tail vein followed TAA injection to verify the function of INF- $\gamma$ to the protective ability of CHI3L1 in the liver. (A) The levels of CHI3L1 with or without INF- $\gamma$ in the serum were determined through ELISA. (B) The levels of INF- $\gamma$ in serum and mRNA expression of Foxp3 in the liver in different groups were determined through ELISA. The serum ALT, AST levels are shown (C), and Suzuki scores were used to evaluate the degree of liver injury (D). Data are shown as means \pm SEM from 3 independent experiments, and each group include 5 mice. NS, no significant; **, $\mathrm{P}<0.01 ;{ }^{* * *}, \mathrm{P}<0.001$.

(Figure 4A). To investigate whether enhanced INF- $\gamma$ in rCHI3L1-treated mice accounted for the balance of Th1/ Treg cells and aggravation of liver injury, INF- $\gamma$ and Foxp3 levels were measured prior to rCHI3L1 and INF- $\gamma$ treatment. The results suggested that $\mathrm{CHI} 3 \mathrm{~L} 1$ signaling relieved TAA-induced liver injury via decreased INF- $\gamma$ factor and improved Foxp3 expression (Figure 4B). Serum ALT and AST levels and Suzuki scores of the livers in the INF- $\gamma$-injected mice were higher than $\mathrm{rCHI} 3 \mathrm{~L} 1$-treated only (Figure 4C,D).

\section{CHI3L1 promotes phosphorylation of the transcription factor STAT-3 via T-bet activation}

T-bet and GATA-binding protein 3 (GATA-3) is reported to have an important regulatory effect on the differentiation of Th1 and Th2 cells $(19,20)$. We investigated whether CHI3L1-KO exerted an effect in the expression levels of T-bet and GATA-3 in vivo depending on the analysis of Th1 and Th2 cells transferred from naive $\mathrm{T}$ cells isolated from $\mathrm{CHI} 3 \mathrm{~L}^{-/-}$mice and their WT littermates. Interestingly, T-bet was strongly expressed in $\mathrm{CHI} 3 \mathrm{L1}^{-/-}$ mice and showed intermediate expression level in WT littermates. GATA-3 was barely expressed, and no differences were observed in Th2 cells (Figure 5A). T-bet activation promoted phosphorylation of the transcription factor STAT-3, which provides essential components involved in the differentiation of Th1 cells. Expression of P-STAT3 in RNA and protein levels were elevated in $\mathrm{CHI} 3 \mathrm{~L}^{1 /-}$ mice derived Th1 cells, which confirmed 

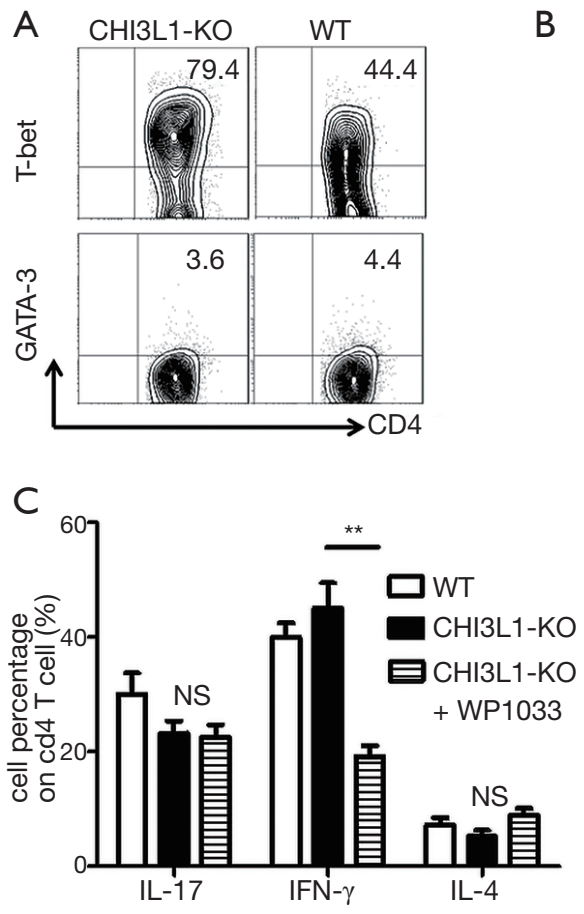

B
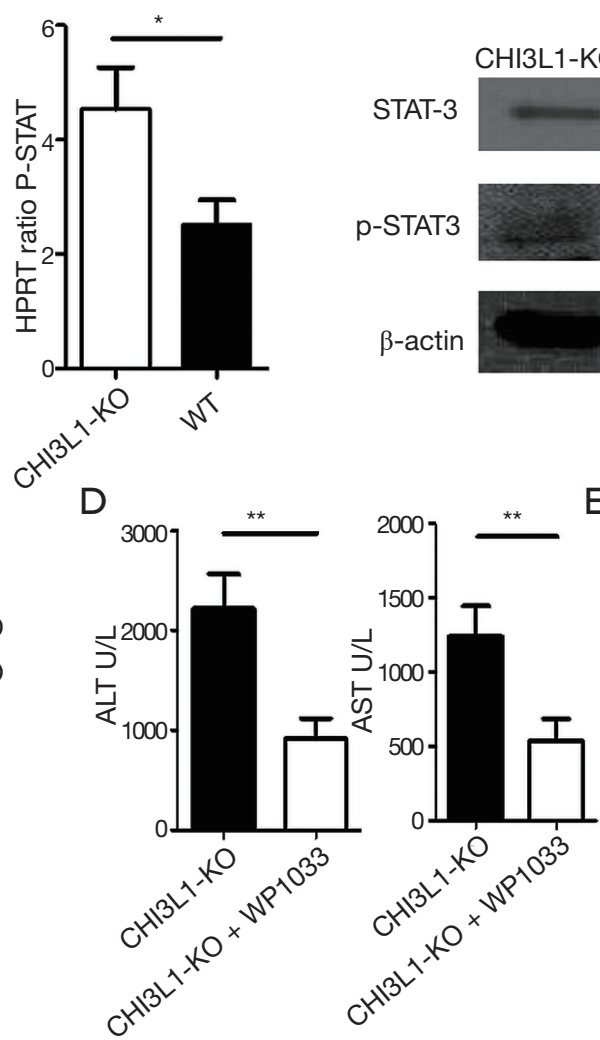
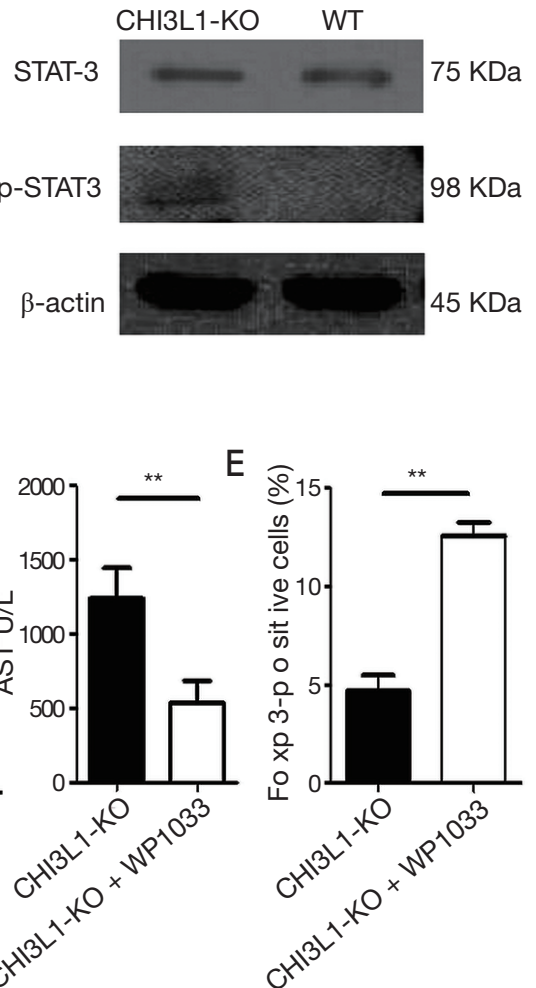

Figure 5 CHI3L1 promotes phosphorylation of the transcription factor STAT-3 via T-bet activation. (A) T-bet and GATA-3 expression in WT and CHI3L1-KO mice were determined by flow cytometry. (B) Expression of P-STAT3 in mRNA and protein levels were analyzed in different groups in ALI models. (C) CD4+CD62L+ T cells extracted from WT and CHI3L1-KO mice were induced to Th17, Th1, and Th2 cells for three days, and WP1033 was added to analyzed the role of STAT3 phosphorylation, the expression of IL-4, IL-17 and IFN- $\gamma$ was detected by flow cytometry. The levels of serum ALT, AST (D), and mRNA expression of Foxp3 (E) in the liver from different groups are shown. Data are shown as means \pm SEM from 3 independent experiments, and for in vivo experiments, each group includes 5 mice. NS, no significant; **, $\mathrm{P}<0.01$; ***, $\mathrm{P}<0.001$. Abbreviations: STAT-3, signal transducer and activator of transcription 3; T-bet, T-box expressed in T cells. GATA-3, GATA-binding protein 3; P-STAT3, phosphorylated signal transducer and activator of transcription 3; WP1033, an inhibitor of P-STAT3.

the hypothesis that $\mathrm{CHI} 3 \mathrm{~L} 1$ promotes phosphorylation of the transcription factor STAT-3 via T-bet activation (Figure 5B). To investigate whether increased levels of P-STAT3 in Th1 cells aggravated TAA-induced liver injury, Th1 cells were treated with P-STAT3 inhibitor WP1006 both in vitro and in vivo. This treatment failed to reduce $\mathrm{IL}-17^{+}$and IL- $4^{+} \mathrm{T}$ cells frequencies, and the proportion of IFN- $\gamma^{+} \mathrm{T}$ cells in $\mathrm{CHI} 3 \mathrm{~L}_{1}^{-/-}$mice-derived naive $\mathrm{T}$ cells was significantly reduced in vitro (Figure 5C). Serum ALT and AST levels in WP1006 treatment CHI3 $\mathrm{L}^{-1-}$ mice were significantly reduced compared to WT littermates (Figure 5D). Given that Treg cells are known to protect from excessive inflammatory-induced liver injury, we examined the expression of Treg cell-specific transcription factors (Foxp3) and found a marked increase in $\mathrm{CHI} 3 \mathrm{~L}^{-/-}$ mice following WP1006 treatment compared to WT littermates (Figure 5E). The combined results indicate that CHI3L1 reduced IFN- $\gamma^{+}$Th1 cell differentiation through inhibiting T-bet/STAT3 signaling pathway and ultimately alleviated liver injury.

\section{Discussion}

Important factors leading to ALI include oxidative stress, excessive inflammatory response, immune cytokine cascade, mitochondrial damage, and centrilobular necrosis $(21,22)$. 
In TAA-exposed rats, pathological observations included unregulated inflammatory response and mass release of proinflammatory cytokines, along with significant mononuclear inflammatory cell infiltration, development of septal and bridging fibrosis, hydropic and fatty degeneration, and enlarged hepatocytes. Controlling this exaggerated inflammatory response and inhibiting the production of proinflammatory cytokines may guide the design of treatment in ALI.

TAA exerts a hepatotoxic effect by the producing free radicals, peroxides, and reactive oxygen species (ROS) (23). ROS accumulation has been reported to produce activated NLRP3 inflammasomes, which promote caspase-1 cleavage, ultimately leading to the production and secretion of proinflammatory cytokines (24). Additionally, activation of NLRP3 inflammasomes has been shown to promote the development of fibrosis in hepatic stellate cells (HSC), with no such effect seen in NLRP3-deficient mice (25). The relationship between CHI3L1 and inflammatory response and associated tissue injury has been well-established in many diseases. In respiratory syncytial virus (RSV)-infected mice, CHI3L1 may enhance airway mucus production, hyperresponsiveness, and inflammatory cell recruitment. RSV-induced airway inflammation and Th2 cytokine production were attenuated in RSV-infected mice treated with anti-CHI3L1 antibody (26). Dong demonstrated that neutrophils' infiltration was inhibited in $\mathrm{CHI} 3 \mathrm{~L}^{-/-}$mice fed an ethanol diet; furthermore, the levels of hydrogen peroxide in the ethanol-fed CHI3L1-KO mice were lower than those in the ethanol-fed WT mice (27). Despite such findings, the role of CHI3L1 in ALI is not fully understood.

To further understand the function of $\mathrm{rCHI} 3 \mathrm{~L} 1$ in ALI, this study investigated $\mathrm{rCHI} 3 \mathrm{~L} 1$ activity in ALI patients and TAA-induced mouse models. The findings revealed that knockout of CHI3L1 produced more severe TAA-induced liver injury, and the administration of $\mathrm{rCHI} 3 \mathrm{~L} 1$ derivatives significantly decreased AST and ALT levels, suggesting that rCHI3L1 derivatives could attenuate TAA-induced liver injury. This was also supported by hematoxylin and eosin staining, which showed reduced necrosis of hepatocytes.

Treg and Th cells are subsets of $\mathrm{CD}^{+} \mathrm{T}$ cells, each with mutually exclusive differentiation and functions (11). IFN- $\gamma$-a signature cytokine of Th 1 cells-can produce IL-2, while IL-4-a signature cytokine of Th2 - can secrete IL-5, IL-6, and IL-13. Cytokine IL-17, which is released by Th17 cells, may induce the accumulation of proinflammatory cells. It is well-established that these
T-cell subpopulations (defined based on their specific transcription factor Foxp3) are involved in ALI Treg cells' progression and are involved in protecting immunemediated ALI through direct contact with cells or release of anti-inflammatory cytokines, such as TGF- $\beta$, IL-10, and IL-35. This study showed that CHI3L1 expression in healthy subjects is almost four times higher than in ALI patients, and its upregulation is important for maintaining the normal function of Treg cells. In contrast, Th cells mediate inflammatory responses and promote immunemediated liver injury by producing cytokines, such as IFN- $\gamma$, IL-2, IL-4, and IL-17 (28).

Interestingly, this study found that Th1 cells may play a greater role in ALI development in CHI3L1-KO mice, compared with Treg cells. Although we observed Treg cells' upregulation and lower proportion of Th cell subsets in $\mathrm{rCHI} 3 \mathrm{~L} 1$-treated mice, this effect was contradicted by the differentiation of Treg, Th2, and Th17 cells between CHI3L1-KO and WT groups, which were smaller. It is speculated that these contradictions may be attributed to poor stability of Treg cells in the inflammatory microenvironment, where proinflammatory cytokines-such as IFN- $\gamma$-were increased dramatically; however, further studies are required to investigate this.

In this study, $\mathrm{rCHI} 3 \mathrm{~L} 1$ exhibited a protective role in TAA-induced ALI mice models by inhibiting IFN- $\gamma^{+}$Th 1 cell differentiation. Further investigation suggested that the expression of IFN- $\gamma$ is upregulated in the liver and circulating lymphocytes in ALI patients. Stimulating the Th1 immune reaction can lead to the activation of CTLs and hepatic macrophages, while the Treg cells attenuate Th1 immunity (29). In the experimental study, where mice were treated with $\mathrm{rCHI} 3 \mathrm{~L} 1$, the association between the immune reactions of CHI3L1 and Th1 cells and liver necrosis was analyzed. This analysis found that CHI3L1deficient $\mathrm{T}$ cells isolated from CHI3L1-KO mice exhibited a higher ability to transfer to Th1 cells and that IFN- $\gamma$ injection reversed the protective function of $\mathrm{rCHI} 3 \mathrm{~L} 1$ treatment, which resulted in serum ALT and AST levels and Suzuki scores due to hepatocyte damage.

Furthermore, the percentage of Treg cells in the liver was significantly depressed by IFN- $\gamma$. Further analysis confirmed the specific role of Th1 cells under the regulation of CHI3L1, showing increased expression of T-bet and enhanced phosphorylation level of STAT-3. T-bet is identified as the lineage transcription factor of Th1 cells and is essential in inducing activated Th cells to differentiate into Th1 cells (30). The T-bet expression is 
sufficient to drive the differentiation of activated naive $\mathrm{Th}$ cells into the Th1 lineage. The findings indicated a novel mechanistic insight into the effect of CHI3L1 on Th1 cell differentiation through the T-bet/STAT3 signaling pathway.

\section{Conclusions}

This study revealed strong anti-inflammatory properties and protective properties in CHI3L1, which combat liver injury pathogenesis. In vivo and in vitro studies revealed that CHI3L1 improved Th1 cell-mediated inflammation in livers of TAA-induced mice by inhibiting IFN- $\gamma$ secretion. Since CHI3L1 is an endogenous factor, these findings may present a promising therapeutic target in developing a nonsurgical ALI treatment.

\section{Acknowledgments}

Funding: This study was supported by the National Natural Science Foundation of China (No. 31700791).

\section{Footnote}

Reporting Checklist: The authors have completed the ARRIVE reporting checklist. Available at http://dx.doi. org/10.21037/atm-20-6127

Data Sharing Statement: The authors have completed the data-sharing statement form. Available at http://dx.doi. org/10.21037/atm-20-6127

Conflicts of Interest: All authors have completed the ICMJE uniform disclosure form (available at http://dx.doi. org/10.21037/atm-20-6127). The authors have no conflicts of interest to declare.

Ethical Statement: The authors are accountable for all aspects of the work in ensuring that questions related to the accuracy or integrity of any part of the work are appropriately investigated and resolved. The study was conducted in accordance with the Declaration of Helsinki (as revised in 2013). The study was approved by the Institutional Review Board of the First Affiliated Hospital of Nanjing Medical University (No. 2015-SRFA-095) and informed consent was taken from all individual participants. All animals received humane care, and experiments were performed under a project license (No. NMU08-
092) granted by the Institutional Animal Care and Use Committee of Nanjing Medical University, in compliance with the US National Institutes of Health guidelines for the care and use of animals.

Open Access Statement: This is an Open Access article distributed in accordance with the Creative Commons Attribution-NonCommercial-NoDerivs 4.0 International License (CC BY-NC-ND 4.0), which permits the noncommercial replication and distribution of the article with the strict proviso that no changes or edits are made and the original work is properly cited (including links to both the formal publication through the relevant DOI and the license). See: https://creativecommons.org/licenses/by-nc-nd/4.0/.

\section{References}

1. Béland K, Marceau G, Labardy A, et al. Depletion of B cells induces remission of autoimmune hepatitis in mice through reduced antigen presentation and help to $\mathrm{T}$ cells. Hepatology 2015;62:1511-23.

2. Manns MP, Lohse AW, Vergani D. Autoimmune hepatitis-Update 2015. J Hepatol 2015;62:S100-11.

3. Robinson MW, Harmon C, O'Farrelly C. Liver immunology and its role in inflammation and homeostasis. Cell Mol Immunol 2016;13:267-76.

4. Liu Z, Meng F, Li C, et al. Human umbilical cord mesenchymal stromal cells rescue mice from acetaminophen-induced acute liver failure. Cytotherapy 2014;16:1207-19.

5. Lee SW, Kim SH, Min SO, et al. Ideal Experimental Rat Models for Liver Diseases. Korean J Hepatobiliary Pancreat Surg 2011;15:67-77.

6. Wallace MC, Hamesch K, Lunova M, et al. Standard operating procedures in experimental liver research: thioacetamide model in mice and rats. Lab Anim 2015;49:21-9.

7. Gujral JS, Farhood A, Bajt ML, et al. Neutrophils aggravate acute liver injury during obstructive cholestasis in bile duct-ligated mice. Hepatology 2003;38:355-63.

8. Bemelmans MH, Gouma DJ, Greve JW, et al. Cytokines tumor necrosis factor and interleukin-6 in experimental biliary obstruction in mice. Hepatology 1992;15:1132-6.

9. Zwacka RM, Zhang Y, Halldorson J, et al. CD4(+) T-lymphocytes mediate ischemia/reperfusion-induced inflammatory responses in mouse liver. J Clin Invest 1997;100:279-89.

10. Niu Y, Liu H, Yin D, et al. The balance between 
intrahepatic IL-17(+) T cells and Foxp3(+) regulatory T cells plays an important role in HBV-related end-stage liver disease. BMC Immunol 2011;12:47.

11. Feng TT, Zou T, Wang X, et al. Clinical significance of changes in the Th17/Treg ratio in autoimmune liver disease. World J Gastroenterol 2017;23:3832-8.

12. Shackelton LM, Mann DM, Millis AJ. Identification of a 38-kDa heparin-binding glycoprotein (gp38k) in differentiating vascular smooth muscle cells as a member of a group of proteins associated with tissue remodeling. J Biol Chem 1995;270:13076-83.

13. Lee CG, Da Silva CA, Dela Cruz CS, et al. Role of chitin and chitinase/chitinase-like proteins in inflammation, tissue remodeling, and injury. Annu Rev Physiol 2011;73:479-501.

14. Ku BM, Lee YK, Ryu J, et al. CHI3L1 (YKL-40) is expressed in human gliomas and regulates the invasion, growth and survival of glioma cells. Int J Cancer 2011;128:1316-26.

15. Singh SK, Bhardwaj R, Wilczynska KM, et al. A complex of nuclear factor I-X3 and STAT3 regulates astrocyte and glioma migration through the secreted glycoprotein YKL40. J Biol Chem 2011;286:39893-903.

16. Johansen JS, Christoffersen P, Møller S, et al. Serum YKL40 is increased in patients with hepatic fibrosis. J Hepatol 2000;32:911-20.

17. Tao H, Yang JJ, Shi KH, et al. The significance of YKL-40 protein in liver fibrosis. Inflamm Res 2014;63:249-54.

18. Malý O, Zajak J, Hyšpler R, et al. Inhalation of molecular hydrogen prevents ischemia-reperfusion liver damage during major liver resection. Ann Transl Med 2019;7:774.

19. Szabo SJ, Kim ST, Costa GL, et al. Pillars article: A novel transcription factor, T-bet, directs Th1 lineage commitment. Cell. 2000. 100: 655-669. J Immunol 2015;194:2961-75.

20. Fang D, Cui K, Hu G, et al. Bcl11b, a novel GATA3-

Cite this article as: Zhang S, Dai X, Shi Y, Zhu X, Dai Y, Qian X, Gu J. CHI3L1 alleviate acute liver injury by inhibiting Th1 cells differentiation through STAT3 signaling pathway. Ann Transl Med 2021;9(7):529. doi: 10.21037/atm-20-6127 interacting protein, suppresses Th1 while limiting Th2 cell differentiation. J Exp Med 2018;215:1449-62.

21. Sánchez-Valle V, Chávez-Tapia NC, Uribe M, et al. Role of oxidative stress and molecular changes in liver fibrosis: a review. Curr Med Chem 2012;19:4850-60.

22. Friedman SL. Mechanisms of disease: Mechanisms of hepatic fibrosis and therapeutic implications. Nat Clin Pract Gastroenterol Hepatol 2004;1:98-105.

23. de David C, Rodrigues G, Bona S, et al. Role of quercetin in preventing thioacetamide-induced liver injury in rats. Toxicol Pathol 2011;39:949-57.

24. Latz E, Xiao TS, Stutz A. Activation and regulation of the inflammasomes. Nat Rev Immunol 2013;13:397-411.

25. Inzaugarat ME, Johnson CD, Holtmann TM, et al. NLR Family Pyrin Domain-Containing 3 Inflammasome Activation in Hepatic Stellate Cells Induces Liver Fibrosis in Mice. Hepatology 2019;69:845-59.

26. Kim MJ, Shim DH, Cha HR, et al. Chitinase 3-like 1 protein plays a critical role in respiratory syncytial virusinduced airway inflammation. Allergy 2019;74:685-97.

27. Lee DH, Han JH, Lee YS, et al. Chitinase-3-like-1 deficiency attenuates ethanol-induced liver injury by inhibition of sterol regulatory element binding protein 1-dependent triglyceride synthesis. Metabolism 2019;95:46-56.

28. Liang $M$, Liwen $Z$, Yun $Z$, et al. The Imbalance between Foxp3(+)Tregs and Th1/Th17/Th22 Cells in Patients with Newly Diagnosed Autoimmune Hepatitis. J Immunol Res 2018;2018:3753081.

29. Leifeld L, Cheng S, Ramakers J, et al. Imbalanced intrahepatic expression of interleukin 12 , interferon gamma, and interleukin 10 in fulminant hepatitis B. Hepatology 2002;36:1001-8.

30. Szabo SJ, Kim ST, Costa GL, et al. A novel transcription factor, T-bet, directs Th1 lineage commitment. Cell 2000;100:655-69. 